\title{
Demonstration of Software-Defined Packet-Optical Network Emulation with Mininet-Optical and ONOS
}

\author{
Bob Lantz ${ }^{1,2}$, Alan A. Díaz-Montiel ${ }^{3}$, Jiakai Yu1 ${ }^{1}$, Christian Rios ${ }^{1}$, \\ Marco Ruffini ${ }^{3}$ and Dan Kilper ${ }^{1}$ \\ ${ }^{1}$ College of Optical Sciences, University of Arizona, Tucson, AZ; ${ }^{2}$ Mininet Project (mininet.org) \\ ${ }^{3}$ CONNECT Centre, Trinity College Dublin, Ireland \\ rlantz@cs.stanford.edu, adiazmon@tcd.ie, jiakaiyu@email.arizona.edu,cdrios@email.arizona.edu, \\ marco.ruffini@tcd.ie,dkilper@optics.arizona.edu
}

\begin{abstract}
We demonstrate practical software emulation of a software-defined, packetoptical network. Our emulator, Mininet-Optical, models the physical, data plane and control plane behavior, under control of the ONOS SDN controller. (C) 2020 The Author(s)
\end{abstract}

OCIS codes: (060.4250) Networks; (060.4510) Optical communications

\section{Overview}

Software-Defined Networking (SDN) control of packet-optical networks has the potential to dramatically improve optical network efficiency, reliability, flexibility, and performance. Disaggregated optical systems, in particular, open the potential for the use of third party optical SDN control and customization [1]. Although hardware testbeds offer ground truth and line rate performance, they are often expensive, hard to reconfigure, and limited in size. The development of innovative optical SDN systems such as ODTN [2] could benefit greatly from a flexible and scalable open source software emulator that emulates the physical, data, and control planes for packet-optical networks [3], much as the Mininet [4] emulator does for packet networks.

To address this need, we have developed Mininet-Optical, which extends Mininet to model optical transmission physics as well as an SDN-controlled optical transmission and switching plane. By integrating optical network modeling with Mininet's existing emulation of IP and Ethernet networks, we can provide a complete platform for testing cross-layer operation of SDN control planes.

For this demonstration, we show how Mininet-Optical can emulate a small network (similar to the COSMOS [5] testbed), as well as larger and more complex topologies such as the well-known Deutsche Telekom topology. We demonstrate optical performance monitoring, impairment-aware wavelength provisioning, and failure recovery in a software-defined, packet-optical network. Mininet-Optical simulates the physical behavior and impairments of the analog optical network, emulates the data plane of both packet and optical networks, and exposes SDN control APIs to ONOS [6], a popular open source SDN control platform used in the commercial Internet.

\section{Innovation}

We demonstrate several new and important capabilities for open source optical network emulation, including:

- End-to-end, interactive emulation of an SDN-controlled packet-optical network

- An emulated optical switching and transmission layer, including a model of the transmission physics

- Discrete emulated optical components such as transceivers, amplifiers, optical fiber spans, and ROADMs

- SDN control and monitoring interfaces for emulated optical network devices

- Scalability to topologies with dozens of optical nodes

- Compatibility with the Mininet emulator for packet networks

- Connecting an emulated packet-optical network to a widely used, open source SDN controller (ONOS) that is deployed in the commercial Internet

\subsection{Design and Implementation}

SDN controllers with optical layer capabilities such as ODTN/ONOS enable new functionality in the optical layer. The ability to evaluate the impact of these controls on the physical performance of the system is important, particularly for large scale systems that approach optical performance limits. The gnpy [7] open source planning tool provides important offline performance prediction of the OSNR/g-OSNR through the GN-based nonlinear interference model. However, it does not support the modeling of SDN control or network emulation. 


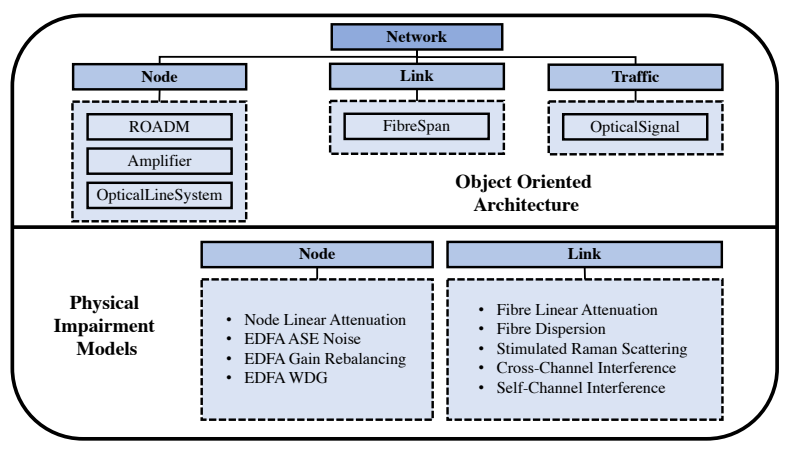

Fig. 1: Mininet-Optical physical-layer architecture and impairment models for the Node and Link objects

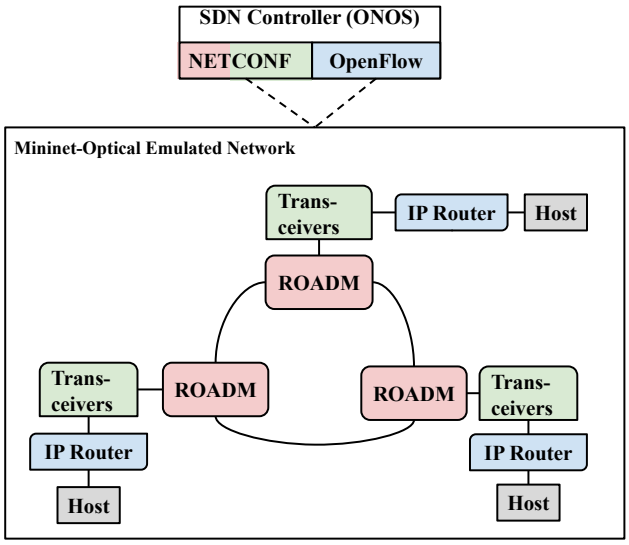

Fig. 2: A simple emulated demo network for packet-optical SDN

Our goal is to combine physical modeling with data plane and SDN control emulation, to enable interactive, end-to-end emulation of an SDN-controlled packet-optical network. This requires a balanced design that is both detailed enough to be useful and efficient enough to be usable and scalable. The current approach is as follows:

1. Physical Modeling: Mininet-Optical abstracts the physical plane using a modular approach to configure optical network devices, such as reconfigurable transceivers, ROADMs with wavelength selective switches (WSSs) and erbium-doped fiber amplifiers (EDFAs) for boost, in-line and pre-amplification, and optical power monitoring (OPM). A simulation subsystem models the optical transmission physics using analytical models (such as the GN-model [8] for fiber nonlinearities) customized to the desired level of detail and implemented in the appropriate devices, as depicted in Figure 1. With this design, each object takes as input the current physical values of the signals in the fiber (modulation, power level, noise levels and wavelength), as well as SDN control state and configuration commands, and computes steady-state output signals that can be easily monitored. A key feature of our implementation design is that it allows customizing the configuration of each network element. For example, users can set wavelength dependent noise figure functions in the EDFAs and input specific wavelength-dependent gain functions, and then evaluate optical transmission systems with signal power fluctuations emulating physical testbed performance. Stochastic parameters can be introduced and result in physical performance that varies with respect to monitored or predicted values.

2. Data Plane Emulation: Mininet-Optical extends packet Mininet by emulating the data plane functionality of optical network elements including optical fiber spans, optical line terminals, transceivers, and ROADMs (including wavelength-selective switches). It models the optical data by augmenting packet data with wavelength/channel information, which can then be transmitted over virtual links and switched by appropriately configured instances of Open vSwitch. Although Mininet-Optical operates at low actual data rates, the physical model provides a simulated data rate and bit error rate, based on encoding and generalized OSNR, for monitoring purposes that corresponds to the behavior of a full-speed hardware network.

3. SDN Control Interface: Mininet-Optical provides an internal SDN control API which is used by external control protocol adapters to provide external control APIs. This design can enable Mininet-Optical to emulate a variety of real-world devices which may use a variety of control protocols (NETCONF, gNMI) and schemata (OpenConfig, OpenROADM [9]). Most importantly, it enables an existing SDN controller, in this case ONOS, to control the emulated network.

\subsection{Example of Operation}

As an example of Mininet-Optical's operation, consider a simple packet-optical ring network topology illustrated in Figure 2. There are 3 network elements connected in this network, each composed of a virtual Host, an IP router, and a ROADM configured with an output port WSS. Each link connecting ROADM nodes is $400 \mathrm{~km}$ long composed of five $80 \mathrm{~km}$ fiber spans with EDFA amplification. Each network element can be configured with the appropriate API (in this example NETCONF or OpenFlow). As in Mininet, users can implement CLI commands that access the control and data plane APIs. Figure 3 demonstrates taking down an optical link, observing a reachability failure, reconfiguring the optical switching layer, and testing for restored connectivity. On the optical monitoring side, the performance (OSNR/gOSNR) of any transmitted optical signal can be recovered from embedded monitors. In Figure 4, $81 \mathrm{C}$-band channels with $50 \mathrm{GHz}$ channel spacing are modulated at 25 GBaud PM-QPSK with -2 dBm launch power per channel. Monitoring results for the optical-signal-to-noise-ratio (OSNR) and generalised-OSNR (gOSNR) are shown for the output ports of each EDFA. 


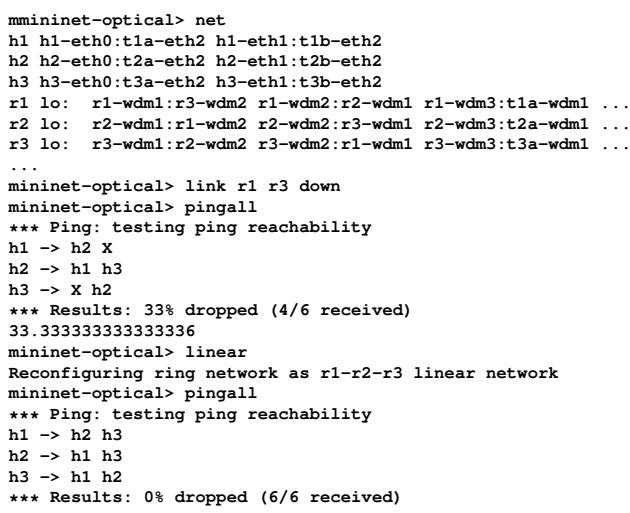

Fig. 3: Example of using the packet-optical control and data plane via the Mininet-Optical console

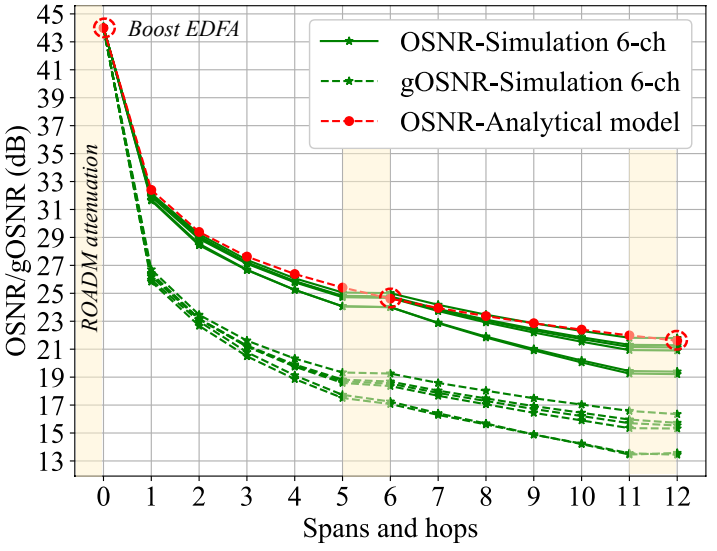

Fig. 4: Example of monitored results from the physical model

\section{OFC Relevance}

This demonstration responds directly to the OFC Demo Zone call for demonstration of software tools to facilitate and accelerate optical SDN research.

In our demonstration, attendees will be able to interact with a live, virtual, optical SDN system, emulated by Mininet-Optical and controlled by ONOS; they will be able to operate the optical SDN control plane (for example adjusting launch power in the transceivers and configuring optical switching in the emulated ROADMs), transmit packets end-to-end across the packet and optical data planes, and monitor modeled physical performance including OSNR/gOSNR and bit error rate.

We view emulation of SDN-controlled packet-optical networks, as with Mininet-Optical, as an essential tool for innovation in the optical networking field, particularly for disaggregated optical systems. It is a key component that will enable a wide range of research, experimentation, development, and education on these systems, empowering researchers, students, and industry practitioners to improve optical networks in new and unexpected ways.

\section{Acknowledgments}

This work is supported by NSF grants \#CNS-1737453 and \#CNS-1827923, the Department of Energy ASCR DE-SC0015867 and the SFI grant 15/US-C2C/I3132.

\section{References}

1. Thyagaturu, Akhilesh S., et al. "Software defined optical networks (SDONs): A comprehensive survey," IEEE Communications Surveys \& Tutorials, vol. 18, no.3, pp. 2738-2786, 2016.

2. Campanella, A., et al. "ODTN: Open Disaggregated Transport Network. Discovery and Control of a Disaggregated Optical Network through Open Source Software and Open APIs," in Optical Fiber Communication Conference, IEEE/OSA, 2019.

3. Díaz-Montiel, Alan A., et al. "Performance analysis of QoT estimator in SDN-controlled ROADM networks," in International Conference on Optical Network Design and Modeling (ONDM), pp. 142-147, IEEE 2018.

4. Mininet (Open Networking Foundation, 2019). http://mininet.org

5. Yu, Jiakai, et al. "COSMOS: Optical architecture and prototyping," in Optical Fiber Communication Conference, pp. M3G-3, IEEE/OSA, 2019.

6. ONOS (Open Networking Foundation, 2019). https : / / nosproject . org/

7. gnpy (Telecom Infra Project, 2019). http: / / pypi .org/project/gnpy/

8. Zefreh, M. Ranjbar, et al. "Accurate Closed-Form GN/EGN-Model Formula Leveraging a Large QAMSystem Test-Set,” IEEE Photonics Technology Letters, vol. 31, no.16, pp. 1381-1384, 2019.

9. Wan, Calvin, et al. "Demonstration of a Software Solution to Support OpenConfig and OpenROADM," in Optical Fiber Communication Conference, pp. M3Z-11, 2019. 